\title{
Evaluación y Determinación de Estándares de Calidad de la Educación Física en la Enseñanza General Básica
}

\author{
Marcelo González Orb, Raúl Santana Núñez ${ }^{\star \star}$, Sergio Guarda \\ Etcheverry $^{\star \star *}$
}

\begin{abstract}
Resumen
Estudio descriptivo, transversal exploratorio, orientado a determinar la calidad de la Educación Física ejercida en las unidades educativas. La muestra tomó como base los establecimientos educacionales del Ministerio de Educación de Chile; del total de establecimientos fueron seleccionados aleatoriamente $\mathrm{n}=44$, los que representan al $3,3 \%$, segmentados en colegios Particular Pagados n=6,2 (8\%); Municipalizados n=2I (5,2\%) y Particular Subvencionados n=I7 (2,2\%). El instrumento "conocimiento reforma educacional" se aplicó a $n=36$, profesores, correspondiendo al $78 \%$ del total, con un missing de $22 \%$. El instrumento "intereses deportivos" se aplicó a n=4.938 alumnos. El estudio permitió determinar el estado del arte respecto a la calidad de la Educación Física ejercida en las unidades educativas, así como las condiciones bajo las cuales se desarrollan éstas.
\end{abstract}

Palabras clave: Gestión; Calidad; Educación; Educación Física.

Evaluation and determination of quality standards for physical education in primary education

\begin{abstract}
Descriptive, transversal and explanatory study targeted at determining the quality of physical education in educational units. The sample included data from educational establishments of the Chilean Ministry of Education; $\mathrm{n}=44$ establishments were selected at random, which represent $3.3 \%$ of the total; they disaggregate into $n=6.2(8 \%)$ paid private schools, $n=2 I$ $(5,2 \%)$ municipal schools, and $n=17(2,2 \%)$ subsidised private schools. The instrument "education reform knowledge" was applied to $n=36$ teachers, representing $78 \%$ of the total, with a missing of $22 \%$. The instrument "sports interests" was applied to $\mathrm{N}=4938$ students. The study determined the state of the art concerning the quality of physical education taught in educational units and the conditions of its development.
\end{abstract}

Key words: Education Administration; Quality; Education; Physical Education.

Doctor de la Universidad Metropolitana de Ciencias de la Educación.

* Magíster de la Universidad Metropolitana de Ciencias de la Educación.

*** Licenciado de la Universidad Metropolitana de Ciencias de la Educación. 


\section{Introducción}

El interés por optimizar en los últimos tiempos la calidad de la Educación y en forma especial la Educación Física, ha sido una temática incisiva especiamente en el ámbito escolar. Sin embargo, pese al avance en el reconocimiento de su trascendencia, se carece de información sobre aspectos que la afectan con el propósito de proponer acciones remediales frente a los puntos críticos.

Si bien el concepto de calidad no marca una postura única, dada su relatividad, es posible considerar un rango evaluativo entre lo ideal, deseable y aceptable, de mala a buena o excelente, condicionándola a ser perfectible. Esta decisión depende de la contrastación frente a patrones establecidos como estándares de calidad. Así, los organismos encargados de velar por la calidad de la Educación en general deben velar por el cumplimiento de estos estándares. Los estándares de calidad se refieren primordialmente a la existencia y grado de preparación del recurso humano que imparte la asignatura, equipamiento, instalaciones e infraestructuras existentes, así como también por su condición de uso otorgado; a los intereses de los escolares en cuanto a sus preferencias deportivas. Dichos estándares se debieran focalizar al logro de resultados de rendimiento eficiente en el sector escolar, sean estos tanto de nivel de la Enseñanza General Básica (EGB) como de la Enseñanza Media (EM).

El modelo de la Reforma Educacional para el Sistema Escolar de EGB y EM que fuera implementado en la década de los 90 bajo el contexto de una sociedad globalizada, dinamizada por la expansión creciente en la utilización del conocimiento, facilitada por el despliegue de modernas tecnologías de la información y comunicación (Cox y García-Huidobro, 1990, 1998), debió adherir al principio que ya para producir competitividad económica como para vivir en comunidad y construir la democracia, se requería de destrezas culturales de nuevo tipo que los sistemas educativos debían comenzar a proveer.

El acento de esta manera se debería orientar a satisfacer los requerimientos formativos, cognoscitivos y morales, pues se trata de evitar aprender cosas aisladas sino de desarrollar capacidades 
y destrezas de aprendizaje, del aprender a aprender, aprender a pensar, aprender a resolver problemas, y aparte de inculcar valores e incrementar la capacidad moral para discernir entre ellos.

Sin embargo, el sistema educativo continúa evidenciando indicadores críticos en cuanto a la calidad de la educación. El problema se centra en su deficiencia de esta variable, la que afecta principalmente a los niños y jóvenes provenientes de grupos más vulnerables, en lugar de poner el acento en los aprendizajes relevantes que efectivamente deberían ser logrados por los estudiantes. Se estimó que una vez mejorada la cobertura, el interés de las políticas se debía trasladar al interior de las instituciones educativas para lograr mayor efectividad de las prácticas pedagógicas. Por lo tanto, los principios orientadores de las políticas educacionales basados en la calidad, debieran exhibir un desplazamiento a los procesos y resultados de aprendizaje.

Se debe tener presente también que el concepto de "reforma" ha estado ligado a modificaciones estructurales, es decir, a cambios de planes y programas de estudio. Empero, si bien se han mantenido los sentidos pedagógicos, se registra escasa atención sobre las formas de enseñar $y$ aprender.

Se esperaba además que el mejoramiento en cuanto a recursos de apoyo educativo, el aumento del tiempo escolar expresado en una jornada única de 8 horas de permanencia dentro del establecimiento y los cambios sustantivos del currículo fueran importantes medios para apoyar el quehacer pedagógico. Los principios para articular distinciones entre contenidos conceptuales, habilidades y disposiciones o actitudes y cambios que permitirían actualizar conocimientos, así como orientar el conjunto de competencias a adquirir mediante experiencias educativas centradas en la actividad de los estudiantes, dio oportunidad para elaborar proyectos educativos propios, pensando en la diversidad y posibilidades de acciones de los establecimientos educacionales.

Las teorías de enseñanza-aprendizaje en base al currículum implantado, mantienen como núcleo básico el concepto de aprendizaje significativo, el cual otorga importancia a la experiencia, al conocimiento previo y al sentido que tiene para los alumnos cada 
concepto o habilidad. De lo expuesto se desprende que, frente al propósito central de una política educativa orientada a mejorar en forma sustantiva la calidad de los aprendizajes de los estudiantes, se pretende disminuir la brecha de inequidad entre escuelas municipales, subvencionadas y particular pagadas.

La reforma curricular de esta manera cambió la Educación Física escolar desde un paradigma de rendimiento centrado en el modelo del deporte de competencias como el ideal de movimiento hacia una educación de exploración de la propia motricidad, reconociendo el movimiento humano como parte del capital cultural de la humanidad.

La orientación propuesta por la Reforma se enlaza de esta forma al concepto de calidad, que proyectada al sector de Educación Física y Deportiva Escolar, puede ser entendida como aquella expresión de la cultura que permite el avance hacia la adquisición de valores, actitudes y hábitos físico-deportivos orientados a una vida activa, saludable y plena, alcanzando desde la propia singularidad logros motores, cognitivos y socio-afectivos. Además, debiera ser efectuada en establecimientos educacionales que disponen de espacios adecuados para la realización de actividades sistemáticas dirigidas por profesionales de la Educación Física y apoyadas con recursos didácticos (MINEDUC, 2002). Esto quedó de manifiesto en la incorporación de ejes temáticos hasta ahora no abordados por las escuelas, tales como los nuevos enfoques de la educación para la vida activa y saludable, la iniciación deportiva desde la perspectiva lúdica, entre otros, los cuales representan un desafío para este sector de aprendizaje.

Es así como a partir de las nuevas orientaciones, se establecieron cuatro criterios generales que orientan las actividades en el sector de aprendizaje: la promoción de la actividad física y un estilo de vida saludable, para lo cual los estudiantes deben ser incentivados a realizar actividad física y participar en actividades que desarrollen su salud; el desarrollo de actitudes personales y sociales positivas, orientando a los estudiantes a una buena conducta deportiva, a un manejo equilibrado de los éxitos y limitaciones, así como valorar el trabajo en equipo; el desarrollo de criterios de seguridad e higiene, 
reconociendo la importancia de aceptar reglas y procedimientos, así como el uso de equipamiento deportivo en la práctica física y la apropiación de los estudiantes del medio natural, sintiéndose a gusto en él y responsables por su cuidado.

Para concretar las aspiraciones educativas del marco curricular, fue necesario apoyar el proceso de implementación y apropiación de dichas directrices, basándose en los ejes curriculares Aptitud física, salud y calidad de vida; Deportes y actividades de expresión motriz; Actividad física en contacto con la naturaleza, con los correspondientes Objetivos Fundamentales (OF), Contenidos Mínimos Obligatorios (CMO) y Objetivos Fundamentales Transversales (OFT). Estos ejes se encuentran orientados hacia la salud, la calidad de vida, hábitos perdurables y con la actitud de vida que transforme a los niños en personas activas y sanas. Como soporte se promulgó la Ley del Deporte (200I), en cuyo articulado se menciona que "el MINEDUC establecerá un Sistema Nacional de Medición de la Calidad de la Educación Física y Deportiva, para ser aplicado al finalizar la EGB, es decir, en $8^{\circ}$ año básico". Fue así como en noviembre del año 2003 se procedió a la aplicación de una prueba piloto de Medición de la Calidad de la Educación Física, la cual arrojó resultados preocupantes en el rendimiento de los estudiantes, corroborando que los riesgos del aumento de sobrepeso y obesidad estaban presentes en más de un tercio de los escolares evaluados. Una de las causantes de las problemáticas visualizadas en la prueba piloto fue referida a la escasa cantidad de horas programáticas de la Educación Física en el sistema escolar, que en promedio persiste en 2 horas semanales. Frente a esta problemática, se han propuesto una serie de iniciativas como el aumento a 4 horas semanales de Educación Física, precisando que la EGB y EM debería considerar al menos 4 horas para el cumplimiento de los planes y programas, destinando así tiempo a la formación deportiva. Otra línea de acción está relacionada al desarrollo del deporte escolar, es decir, establecer estrategias en conjunto con el MINEDUC para la creación y desarrollo de Clubes Deportivos Escolares. Además, establecer programas focalizados al desarrollo psicomotor para niños y jóvenes en edad escolar, incentivar el cumplimiento de los objetivos fijados en planes y crear programas de orientación y de estimulación psicomotriz a edad temprana. 
Entonces cabría preguntarse: ¿cómo entender la calidad de la Educación Física en la EGB?

Es indudable que entre las variables influyentes en la calidad de la Educación Física escolar, las más significativas serían: la preparación específica del profesorado, la infraestructura y materiales que la asignatura requiere, así como el contemplar los intereses deportivos de los estudiantes, contrastando la oferta deportivo-recreativa que entregan los establecimientos, entre otros.

En lo que respecta a los profesores de Educación Física, la capacitación es vital para la creación de hábitos destinados a promover el ejercicio físico-deportivo. Resulta fundamental propiciar el perfeccionamiento con metodologías de enseñanza actualizada como base del proceso de motivación. La Reforma Educacional propone el manejo de contenidos mínimos obligatorios por parte de los docentes, factor muy importante ya que quienes tienen la responsabilidad de llevar a cabo las actividades, omiten elementos claves tales como la organización y evolución jerárquica de los contenidos a aplicar.

Por otra parte, los profesionales de la actividad física deben contar con recursos materiales, razón que hace necesario considerarlos como elementos facilitadores del logro de los aprendizajes en función de la cantidad requerida, ajustados a las instancias etarias, al número y características de los estudiantes a los cuales se dirigirá la clase, entre otras. En algunas oportunidades se observa a los docentes exigir materiales y por lo general los directivos no atienden esta necesidad. No obstante, hay que tener siempre presente que el uso inadecuado de algunos implementos puede provocar lesiones o carencia de motivación, aspecto destacado por Sánchez y Román (2005).

Sin embargo, la infraestructura y dotación de material son imprescindibles, pues afectan directamente el quehacer pedagógico. Contar con ellos resulta esencial para el éxito del proceso, de tal manera que dotar a los establecimientos adecuadamente de éstos otorga señales claras para cumplir con eficacia el quehacer pedagógico. En consecuencia, resulta obvio que es responsabilidad de quien esté al frente de la organización educacional, sea el Estado o entidad privada, aportar las herramientas tanto en cantidad y calidad. 
Entonces, ¿cómo establecer la realidad presente de la Educación Física en la EGB?

Estudios desarrollados por González et al. (2005-2009), junto con diseñar, validar y aplicar baterías de instrumentos destinados a evaluar los agentes incidentales en la calidad de la Educación Física, han arribado a conclusiones no muy alentadoras frente a los resultados esperados en esta asignatura, pues dejan en evidencia la realidad de colegios chilenos de EGB. Al respecto, se logró establecer diferencias significativas en relación a la inequidad en todos los rubros anteriormente mencionados.

Así, se aprecian inequidades entre la Educación Física impartida en las distintas modalidades educacionales. Si bien la brecha es amplia en materia de infraestructura y en cantidad de recursos materiales, lo más preocupante es el nivel de dominio de contenidos mínimos obligatorios de quienes tienen la responsabilidad de estar al frente del desarrollo de la asignatura. Es indudable que los antecedentes recogidos que dan cuenta del estado real del proceso formativo, deben ser seriamente considerados como evidencias que permitan la toma de decisiones futuras para un mejor desenvolvimiento de la Educación Física. Contar con información actualizada permite establecer atinadamente cuán próximo o distante se encuentra esta práctica respecto a los estándares de calidad, básico para introducir mejoras y facilitar el aprovechamiento de los recursos humanos y materiales.

Pero ¿qué es calidad?, ¿qué se entiende por estándar de calidad?, ¿cuáles son los estándares o parámetros que se consideran óptimos, en lo referido a la Educación Física? Un estándar de calidad es el requisito óptimo de excelencia, por ende se entenderán como tal, aquellas exigencias que lleven a la excelencia. Es un soporte de gran importancia para obtener resultados sobresalientes, por lo que resulta clave entender y establecer cuáles son estos estándares o parámetros. El concepto de "calidad", más que ser un cuestionamiento teórico, es parte de un ineludible compromiso personal de poner a disposición las herramientas para facilitar una tarea. En la tarea se consideran aspectos y criterios, los cuales se fundamentan en objetivos específicos, es decir, a lo que se desea llegar y con esto 
estimar un sistema de educación de calidad. Esta condición exige realizar evaluaciones continuas que ayuden a ejercer las decisiones con precisión y efectividad para provocar los cambios requeridos en pro de la optimización de los procesos educativos.

Surgen de esta manera nuevas interrogantes: ¿cuál es el estándar de la calidad de la Educación Física, a nivel de EGB?, ¿cuál es el nivel de conocimiento por los profesores de EGB de los contenidos mínimos de la reforma en materias de Educación Física?, ¿existe correlación respecto a los intereses físico-deportivos de los alumnos de la EGB y las actividades desplegadas en las clases de Educación Física?, ¿el equipamiento utilizado en las clases de Educación Física es adecuado y de calidad? A partir de estas premisas se derivan los siguientes objetivos relacionados a la investigación:

I. Determinar la calidad de la Educación Física desarrollada en establecimientos educacionales Municipales, Particulares Subvencionados y Particulares Pagados de EGB ( $2^{\circ}$ a $8^{\circ}$ año).

2. Establecer el nivel de conocimiento que poseen los profesores en cuanto a programas del área de Educación Física para la EGB y contenidos mínimos de cada nivel planteados por el MINEDUC.

3. Determinar la relación oferta de actividades físico-deportivas versus intereses deportivos-recreativos de los alumnos de los establecimientos seleccionados.

4. Establecer las condiciones en las cuales se desenvuelve la práctica físico-deportiva, infraestructura y equipamiento de los establecimientos educacionales.

\section{Material y método}

El estudio descriptivo, transversal y exploratorio se orienta a determinar la calidad de la Educación Física ejercida en las unidades educativas y establecer las condiciones de cómo se desarrolla ésta, con el propósito de proyectar los hallazgos y aportar información destinada a optimizar su desarrollo. 


\section{Población y muestra}

La muestra está determinada por los siguientes criterios: pertenecer a zona urbana, corresponder a establecimientos educacionales de EGB completa y ser de dependencia municipal, particular pagada y particular subvencionada.

El marco muestral tomó como base el directorio de establecimientos educacionales del Ministerio de Educación de Chile. El total de establecimientos corresponde a $\mathrm{N}=\mathrm{I} 370$, comprendiendo a $\mathrm{n}=2 \mathrm{I} 0$ particulares pagados, $n=400$ municipales, $n=760$ particulares subvencionados. Del total fueron seleccionados aleatoriamente $\mathrm{n}=44$, los que representan al 3,3\%, de los cuales los segmentos educacionales quedaron representados de la siguiente manera: particular pagado $n=6,(3,5 \%)$, municipalizados $n=2 I(5,2 \%)$, particular subvencionado $\mathrm{n}=\mathrm{I} 7(2,2 \%)$. Los instrumentos sobre intereses deportivos, fueron aplicados a $\mathrm{n}=4.938$ alumnos, 20 alumnos por nivel, de $2^{\circ}$. a $8^{\circ}$. año básico (I0 mujeres y 10 hombres). El instrumento sobre nivel de conocimiento de la reforma educacional se aplicó a $n=36$ profesores, correspondiendo al $78 \%$ del total, existiendo un número no considerado (missing) de $22 \%$, debido a razones de ausencia o negación a ser evaluados.

\section{Métodos cuantitativos}

\section{Instrumentos}

En el desarrollo del estudio se tomó como base los instrumentos mencionados para la determinación de estándares de calidad de la Educación Física en la EGB elaborados por González et al. (2008), los cuales permiten determinar el nivel de conocimientos de planes y programas por parte de los profesores, establecer la calidad de la infraestructura y material didáctico utilizado e intereses deportivos de los alumnos.

Para establecer la calidad de la Educación Física, esto es, nivel de conocimiento de los profesores respecto a planes y programas, se sumaron las puntuaciones obtenidas en cada dimensión. Para 
establecer el perfil global de calidad, el protocolo contempló dos procedimientos: visitas a establecimientos y aplicación de instrumentos en forma directa. La validez de contenido se obtuvo mediante juicio de expertos, en concordancia con el modelo teórico. La validez de constructo se gestó mediante análisis factorial, realizando ajustes (reasignación de ítems y dimensiones) en cada uno de ellos. La fiabilidad fue establecida respetando las sugerencias de Gómez (1997), Hernández, Fernández, y Baptista (2003). La consistencia interna se obtuvo mediante el coeficiente Alfa de Cronbach (195I), teniendo como criterio considerarlo aceptable cuando resulta igual o superior a 0.70 .

El instrumento "nivel de conocimiento por profesores" posee una fiabilidad de ,704 lo cual es un puntaje medianamente aceptable para este tipo de instrumentos.

\section{Estadísticos de fiabilidad}

\begin{tabular}{|r|r|}
\hline $\begin{array}{c}\text { Alfa de } \\
\text { Cronbach }\end{array}$ & $\begin{array}{c}\text { N de } \\
\text { elementos }\end{array}$ \\
\hline, 704 & 40 \\
\hline
\end{tabular}

\section{Resultados}

Análisis por pregunta, nivel de conocimiento planes y programas de Educación Física, MINEDUC.

Respecto a los ítems que presentan mayores respuestas fallidas o incorrectas se encuentran:

-¿Qué entiende por habilidades motrices básicas? Del total de profesores que respondieron $n=32$, lo que representa el $100 \%$, el $62,5 \%$ contestó incorrectamente;

-¿Qué entiende por destreza motriz? De n=32 profesores, 2I,70\% respondió de forma incorrecta;

-¿Qué entiende por aptitud física? Un 82,8\% respondió de forma incorrecta;

- ¿Cuál es la progresión para pasar de un juego a un deporte? De $\mathrm{n}=32$ profesores, el 93,5\% marcó la opción de forma incorrecta; 
- ¿Cuál es la diferencia entre la técnica de un deporte y la táctica del mismo? De n=3I profesores el 100\% respondió de manera incorrecta;

- ¿Cuál de las siguientes alternativas no está dentro de las técnicas de expresión corporal? De n $=28$ profesores, el 60,7\% marcó la opción de forma incorrecta;

-¿Cuál de los siguientes deportes corresponden a deportes de expresión rítmica motriz? Un 78, I\% respondió de forma incorrecta;

-¿Cuáles son los principios generales del juego? De $\mathrm{n}=28$ profesores, el 64,3\% marcó de forma incorrecta;

- ¿Cuáles son los principios de entrenamiento de la Aptitud Física? De $\mathrm{n}=28$ profesores, los que respondieron de forma válida, un $96,4 \%$ la respondió de forma incorrecta;

-¿Qué habilidades sociales colaborativas se encuentran presentes en el básquetbol? De n=3I profesores, el I00\% respondió incorrectamente;

-¿Qué pasa en caso de que el marcador al final de un partido de Básquetbol el resultado es empate? De $n=30$ profesores, un $63,3 \%$ marcó incorrectamente;

- ¿Cuál de los siguientes elementos es una conducta de entrada para realizar un pase dirigido? Del total $n=3 \mathrm{I}$ profesores, un $6 \mathrm{I}, 3 \%$ marcó de forma incorrecta;

-¿Cuál de los siguientes elementos corresponde a un elemento técnico del Vóleibol? Del total $n=22$ profesores, un 95,5\% lo contestó de forma incorrecta;

-¿Cuál de las siguientes situaciones está permitida realizar durante un partido de Hándbol? De n=29, un 86,2\% respondió de forma incorrecta;

-¿Qué conducta de entrada debe tener un alumno antes de enseñarle la rueda? Un 74,2\% marcó la opción incorrecta;

- ¿Cuál de las siguientes situaciones no se recomienda realizar al acampar? Del total n=24, un 91,7\% marcó la opción incorrecta; 
-¿Cuál de las siguientes alternativas no sirve como un medio de orientación? De $n=29$ docentes, un $89,7 \%$ marcó la opción incorrectamente;

-¿Cuál es la progresión metodológica del nado crol? De n= $3 \mathrm{I}$ docentes, un $83,9 \%$ marcó de forma incorrecta;

-¿Qué recomendaciones se deben tener en cuenta en natación como medida de seguridad de los alumnos? De $\mathrm{n}=30$ docentes, un I00\% marcó de forma incorrecta;

-¿Cuál es el principio fundamental de salvataje? De $n=29$ docentes, un $65,5 \%$ marcó la opción de forma incorrecta;

-¿Cuáles son las fases principales de una clase de Educación Física? De $\mathrm{n}=28$ docentes, el 92,9\% marcó de forma incorrecta;

-¿Qué se entiende por evaluación sumativa y formativa? De $n=26$ docentes, un $80,8 \%$ marcó de forma incorrecta.

\section{Análisis por dependencia}

Consideró las preguntas correctas e incorrectas de cada profesor encuestado, según dependencia: establecimiento municipal, particular pagado y particular subvencionado.

Gráfico $\mathrm{N}^{\circ} \mathrm{I}$. Establecimientos Municipales

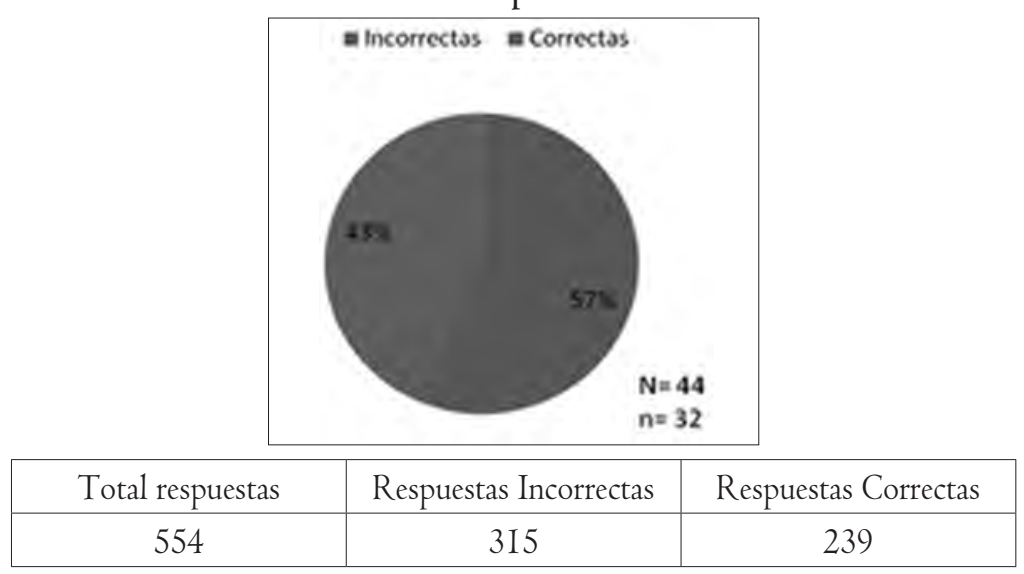


Los profesores de los establecimientos Municipales obtuvieron un porcentaje de $56,9 \%$ de respuestas incorrectas, entrando en la denominación de malo.

Gráfico N². Establecimientos Particulares Pagados

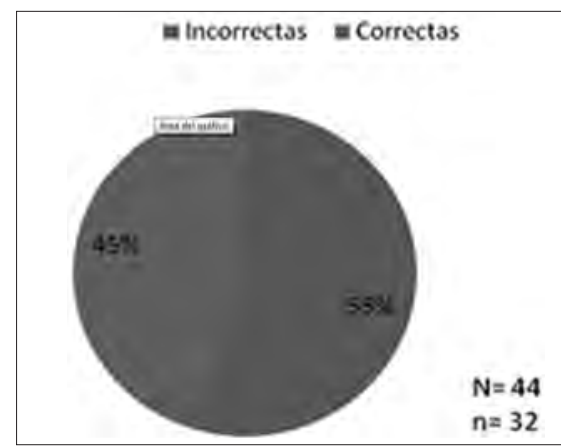

\begin{tabular}{|c|c|c|}
\hline Total respuestas & Respuestas Incorrectas & Respuestas Correctas \\
\hline I66 & $9 \mathrm{I}$ & 75 \\
\hline
\end{tabular}

Los profesores de los establecimientos Particulares Pagados, un porcentaje de $54,8 \%$ entran en la denominación de malo.

Gráfico No3. Establecimientos Particulares Subvencionados

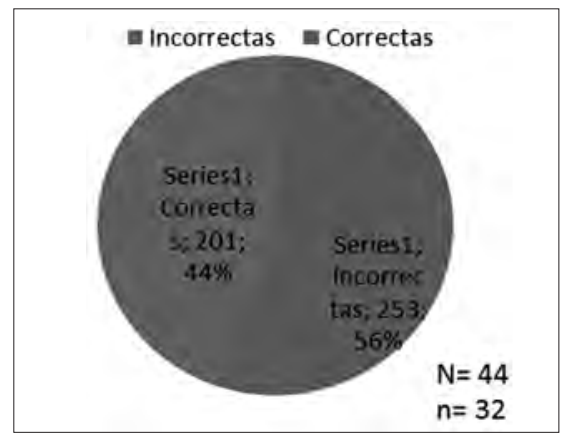

\begin{tabular}{|c|c|c|}
\hline Total respuestas & Respuestas Incorrectas & Respuestas Correctas \\
\hline 454 & 253 & 201 \\
\hline
\end{tabular}

Los profesores de los establecimientos Particulares subvencionados, que obtuvieron un porcentaje de $55,7 \%$ entran en la denominación de malos. 


\section{Análisis general}

Es significativo destacar que de un total de 40 , fueron respondidas 22 preguntas de forma incorrecta $(55 \%)$.

La mayor cantidad de respuestas erróneas se presenta en fundamentos técnicos generales, que se basan principalmente en contenidos de deportes. Por lo que evidencia que estos contenidos se están impartiendo de forma incorrecta en el alumnado.

Los siguientes porcentajes de profesores de los establecimientos Municipales (57\%), Particulares Pagados (55\%), y Particulares Subvencionados (56\%), entran en la denominación de malo, con lo que es posible determinar que la dependencia económica de cada establecimiento no influye en la calidad de sus docentes, ya que el porcentaje de variabilidad entre ellos es mínimo.

Por otra parte el análisis comparativo por dependencia permite indicar que los establecimientos Municipales fueron los que obtuvieron mayor cantidad de respuestas incorrectas, seguido por los establecimientos Particulares Subvencionados y finalmente los establecimientos Particulares Pagados.

\section{Análisis Instrumento Evaluación de Infraestructura}

El instrumento fue aplicado en un total de $\mathrm{N}=44$ establecimientos de distinta dependencia, $n=2 I$ establecimiento Municipales, $n=6$ establecimientos Particulares Pagados, y $\mathrm{n}=\mathrm{I} 7$ establecimientos Particulares Subvencionados.

Los establecimientos Particulares Pagados presentan mayor existencia de infraestructura ligada a la práctica física. Los establecimientos Municipales poseen instalaciones básicas como patio techado, sala multiuso y juegos infantiles.

Respecto a la antigüedad de construcción, los que presentan mayor antigüedad son los establecimientos Particulares Subvencionados con un 46,7\%, mientras los establecimientos Municipales y Particulares Pagados asumen modernización en un 25\% y 20\% respectivamente. 
En cuanto a modificaciones de las instalaciones desde su construcción, los establecimientos Municipales son los que manifiestan mayores modificaciones con un 82,3\%, mientras los Particulares Subvencionados un 60\% y los Particulares Pagados un $75 \%$, lo que evidencia que se están realizando cambios en las estructuras deportivas, con el fin de cubrir las necesidades.

¿Dónde se realizan las modificaciones? Las mayores modificaciones de establecimientos Municipales se han realizado en patios y multicanchas. En los establecimientos Particulares Pagados, han sido realizadas en los patios, gimnasios, y multicanchas. Finalmente, para los establecimientos Particulares Subvencionados, principalmente se observan modificaciones en patios, multicanchas y gimnasios. Por lo tanto, las instalaciones de mayor uso, desgaste o necesidad en los distintos establecimientos son los patios, multicanchas y gimnasios.

Gráfico ${ }^{\circ} 4$. Modificación en instalaciones deportivas.

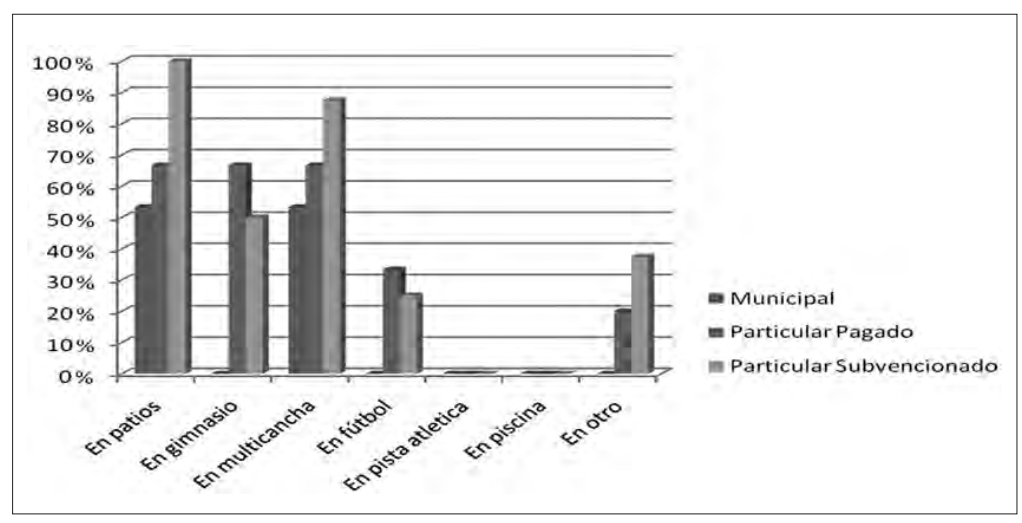

Respecto a la existencia de estructuras adicionales a las instalaciones deportivas, los establecimientos Municipales las poseen en un $33,3 \%$ a diferencia de los establecimientos Particulares Pagados y Subvencionados, elevados a valores de $75 \%$ y $80 \%$ respectivamente.

En relación a arriendo de instalaciones, los establecimientos Municipales no arriendan sus instalaciones deportivas, mientras los establecimientos que tienen un porcentaje mayor de arriendo son los Particulares con un 50\%, seguidos de los Subvencionados con un $33,3 \%$. 
En referencia a la limpieza de las instalaciones después de cada clase, los establecimientos Particulares manifestaron un I00\% en la respuesta sí, frente a un 73,3\% de los establecimientos Particulares Subvencionados y en último lugar los establecimientos Municipales con un $60 \%$ de respuesta afirmativa.

En la existencia de planes de mantención y conservación de las instalaciones, el 50\% de los establecimientos Municipales y Particulares Pagados manifiestan poseerla, mientras los Subvencionados solo en un I4,3\%.

En relación a la mantención durante vacaciones, la dependencia que obtuvo la mayoría en respuesta afirmativas, son los establecimientos Particulares Subvencionados, seguidos de los Municipales y en último lugar los Particular Pagado con un $75 \%$.

Por otra parte, en relación a la generación de instalaciones nuevas, los que presentan mayor porcentaje son los establecimientos Particulares Pagados, mientras los Municipales manifiestan un 3I,3\%, los Particulares Subvencionados solo manifiestan un I4,3\% de creación de nuevas instalaciones.

En cuanto a la realización de modificaciones en los últimos cinco años, los establecimientos que han realizado más son los Particulares Pagados (100\%), seguido de los Municipales (52,1\%) y Particulares Subvencionados con un $42,9 \%$.

En referencia al lugar de realización de las actividades deportivas, se puede señalar que estas varían de acuerdo a la modalidad educacional, lo cual se puede apreciar en el gráfico precedente, en que las clases en establecimientos Municipales son principalmente realizadas en multicanchas y patios, mientras los establecimientos Particulares Pagados, en instalaciones como el gimnasio, cancha de fútbol y otras. Por último, en los establecimientos Particulares Subvencionados éstas son realizadas en gimnasio, multicanchas y patios. 
Gráfico $\mathrm{n}^{\circ} 5$. Lugar de realización de las clases de educación física.

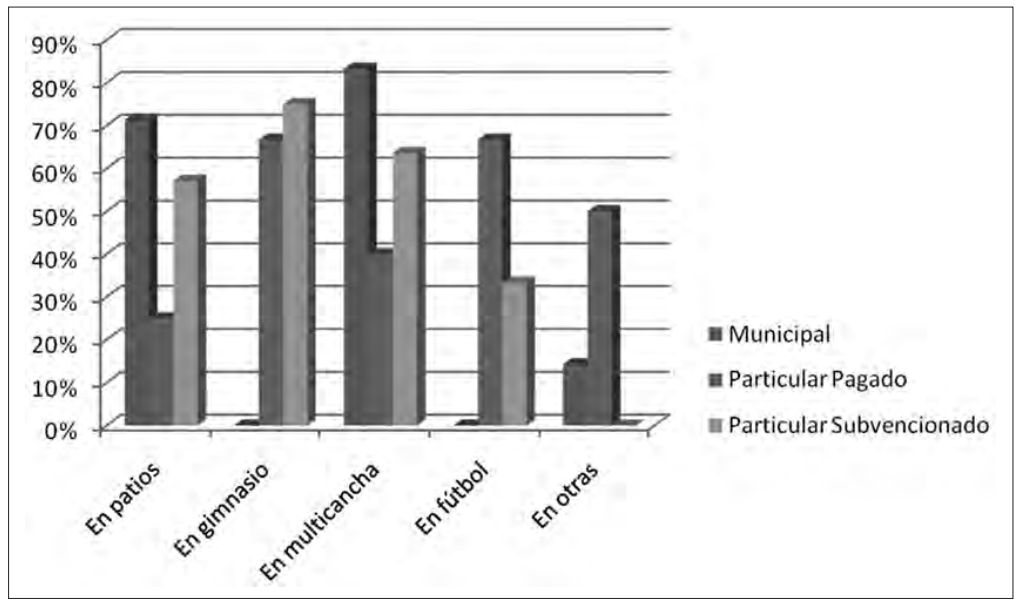

\section{Análisis General}

Considerando la infraestructura, es posible señalar que los colegios Particulares Pagados aventajan notoriamente a los establecimientos Municipales y Particulares Subvencionados. Los Particulares Pagados evidencian mayor existencia de infraestructura ligada a la práctica de educación física, los establecimientos Municipales, por su parte, manifiestan poseer instalaciones básicas como patio techado, sala multiuso, y juegos infantiles, en su mayoría.

\section{Análisis de Intereses deportivos:}

De acuerdo a los resultados es posible señalar que existe coincidencia en los intereses deportivos de los alumnos de establecimientos Municipales y Particulares Subvencionados, las tres primeras preferencias para ambos son: fútbol con primera mayoría, ciclismo con segunda mayoría para los Municipales y tercera mayoría para los Particulares Subvencionados y natación como tercera opción para los Municipales y segunda para los Particulares Subvencionados.

Los Particulares Pagados coinciden en cuanto al interés por natación y ciclismo, siendo la primera de mayor preferencia por los alumnos 
y el ciclismo es la tercera preferencia. La segunda preferencia es el tenis para los alumnos de este tipo de dependencia.

En relación a la oferta deportiva es importante destacar que en los establecimientos Municipales y Particulares Subvencionados el único interés de los alumnos que coincide con la oferta deportiva del colegio es el fútbol (dentro de las tres primeras mayorías). En los establecimientos Particulares Pagados no existen relaciones claras en cuando a la oferta y los intereses. De todas formas, es necesario destacar que la opción que sugiere otros deportes no explicitados resulta altamente indicada en este tipo de establecimientos.

Gráfico ${ }^{\circ} 6$. Promedio Intereses deportivos según dependencia.

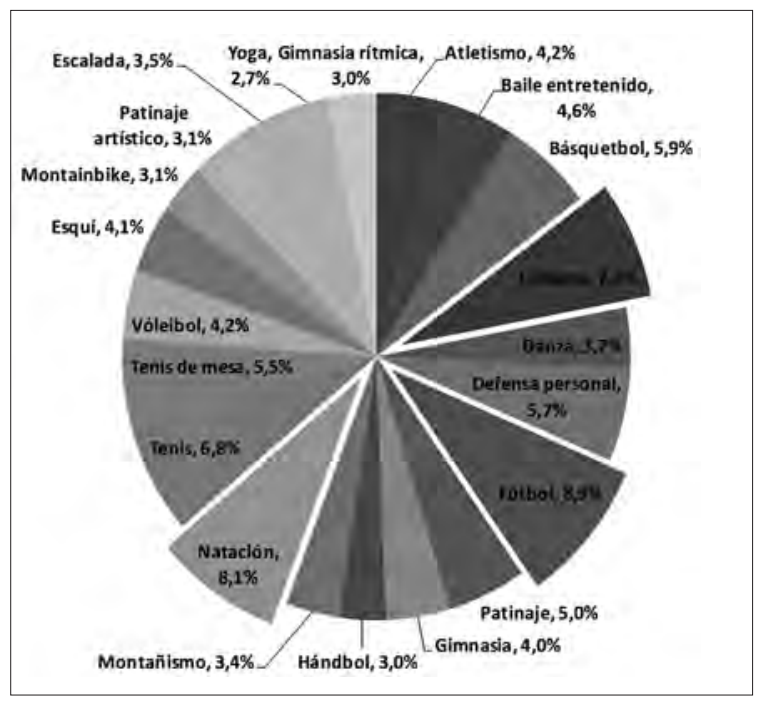

En referencia al promedio de Intereses deportivos, las preferencias de los tres tipos de establecimientos indican que, en orden decreciente, las primeras cinco son fútbol, natación, ciclismo, tenis y defensa personal.

Respecto a la utilización del material didáctico, se dan variaciones significativas en los tres tipos de establecimientos. Los profesores de establecimientos Municipales sí utilizan material didáctico, pero este sólo "a veces", de acuerdo a la cantidad de alumnos. 
Gráfico $\mathrm{n}^{\circ}$ 7. Cantidad material didáctico según naturaleza establecimiento.

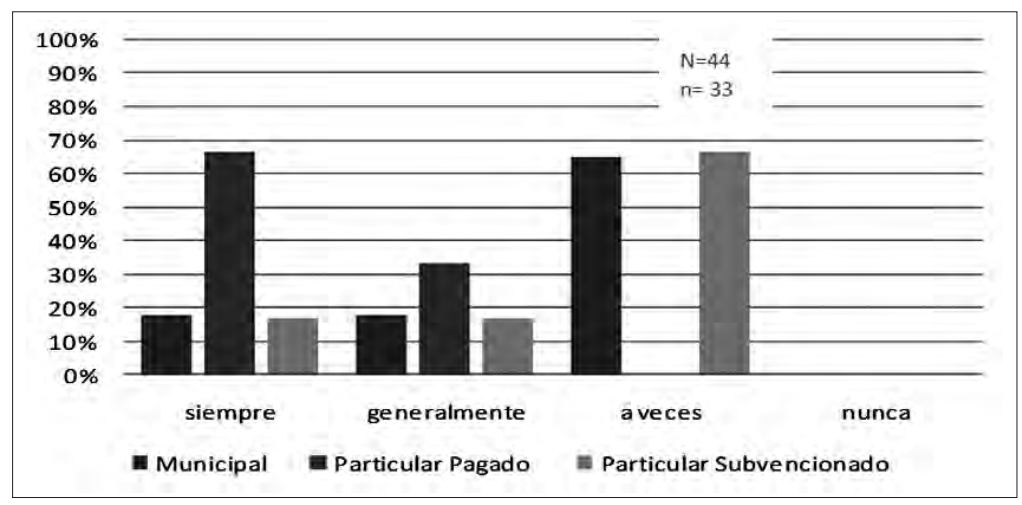

En cuanto a la renovación y mantención del material, ésta se efectúa esporádicamente, ya que el criterio para la inversión en material está dado por los recursos económicos del establecimiento, los cuales los profesores consideran insuficientes. En el caso de los establecimientos Particulares pagados, los profesores siempre utilizan el material didáctico en sus clases, estando acorde a la cantidad de alumnos. Los recursos destinados al material didáctico son elevados en cuanto a la mantención y renovación, con mayor preferencia por la mantención de estos. Los establecimientos Particulares Subvencionados utilizan "siempre" el material didáctico en las clases, aunque sólo "a veces" éste se encuentra en función del número de alumnos.

Respondiendo a la fuente de adquisición, los establecimientos Municipales manifiestan que la principal fuente es de los propios establecimientos. Según la disponibilidad del material, sólo los establecimientos Particulares Pagados disponen totalmente de estos; los establecimientos Particulares Subvencionados y Municipales figuran con bajos porcentajes. La inversión de material didáctico en los establecimientos Municipales y Subvencionados es escaso y solo los Particulares Pagados consideran suficiente la inversión.

Revisando la práctica de deportes después de la escuela hay diferencias significativas en cuanto al tipo de establecimiento. Los alumnos de establecimientos Particulares Pagados son los que más practican deporte después de la escuela mientras los de establecimientos Municipales son los que menos practican. Indagando el nivel de 
interés demostrado por la práctica deportiva, son los 4os. años básicos los que más afición tienen por ella. De la misma forma se registra una baja considerable en los 7os. y 8os. años básicos a esta afición.

\section{Discusión}

Acorde a los resultados obtenidos, es posible inferir que en relación a la calidad de la educación, no hay concordancia con lo formulado en los modelos teóricos, es decir, la calidad de la Educación Física desarrollada en los establecimientos educacionales Municipales, Particulares Subvencionados y Particulares Pagados de la Provincia de Santiago, de EGB $\left(2^{\circ}\right.$ a $8^{\circ}$ año $)$ se puede considerar como regular. El nivel de conocimiento que poseen los profesores de los programas del área de Educación Física (EGB), de los contenidos mínimos planteados por el MINEDUC, no concuerdan con lo esperado en referencia al saber teórico de los profesores y a la oferta de actividades físico-deportivas e intereses deportivos-recreativos. También se manifiesta una discrepancia entre el contexto teórico y la realidad referida a la motivación, en las condiciones en las cuales se desarrolla la práctica físico-deportiva, infraestructura y equipamiento. En los establecimientos educacionales tampoco se cumple con el deber ser de lo planteado en el modelo teórico con lo existente.

\section{Conclusiones}

El nivel de conocimiento que poseen los profesores de los programas de Educación Física (EGB), los contenidos mínimos de cada nivel del MINEDUC, fueron evaluados en la clasificación de "malo", con errores similares y frecuentes entre los tres tipos de dependencias educacionales.

Se debe destacar el escaso interés de los profesores por participar en los procesos de evaluación, siendo posible inferir que estos no se sienten seguros de sus conocimientos o simplemente no están comprometidos con introducir mejoras en la asignatura.

Respecto a la propuesta de actividades físico-deportivas versus inclinaciones deportivas-recreativas de los estudiantes, es posible 
inferir que existe escasa concordancia entre oferta e intereses, respectivamente.

A partir de la información obtenida, se desprende que es preciso ampliar la gama de oferta, potenciando actividades deportivas como natación, la que cuenta con una alta demanda en las tres dependencias.

Se debe destacar que los establecimientos Particulares Pagados denotan una demanda más amplia de intereses deportivos, tanto en mujeres como hombres, al parecer por contar con más implementación e infraestructura como son natación, gimnasia rítmica y esquí, las que se encuentran dentro de las tres primeras preferencias que suelen ser cubiertas mediante uso de dependencias externas o bien con la inclusión de programas establecidos para concurrir por ejemplo a esquiar en fecha invernal.

En referencia a la infraestructura existente en los establecimientos de las tres dependencias, es posible inferir que los Particulares Subvencionados y Municipales cuentan sólo con infraestructura básica (multicanchas y patios), las cuales además de no estar siempre disponibles, se encuentran en condiciones impropias para la práctica de los deportes con mayor demanda. Por el contrario, los Particulares Pagados cuentan con instalaciones aptas, en buenas condiciones y con disponibilidad suficiente para el desarrollo de la práctica deportiva con más demanda.

En referencia al aspecto material didáctico deportivo, es posible señalar que la tendencia mostrada por los establecimientos Municipales y Particulares Subvencionados, sólo a veces se encuentra acorde a la cantidad de alumnos, siendo por lo general insuficiente y poco adecuado para realizar talleres y actividades que satisfagan los intereses de los alumnos. Por el contrario los Particulares Pagados cuentan con material adecuado de mejor calidad y acorde al número de alumnos, por lo que en estos establecimientos se desarrolla una mayor gama de actividades y talleres extraprogramáticos, satisfaciendo la demanda de los alumnos.

Observando las condiciones en las cuales se desarrolla la práctica físico-deportiva (infraestructura), los establecimientos Municipales 
cuentan con las instalaciones básicas y en condiciones regulares, al igual que los establecimientos Particulares Subvencionados. Los establecimientos Particulares Pagados evidencian significativamente mayor y mejor infraestructura deportiva.

Finalmente, en cuanto a la calidad de la Educación Física desarrollada en los establecimientos Municipales, Particulares Subvencionados y Particulares Pagados de EGB $\left(2^{\circ}\right.$ a $8^{\circ}$ año) de la provincia de Santiago, es posible señalar que según los parámetros de evaluación para las dependencias, conocimiento de planes y programas, material didáctico, infraestructura e intereses deportivos, los Particulares Pagados son los que se aproximan al concepto de "calidad", seguidos en mayor distancia por los Particulares Subvencionados y a la zaga quedan los establecimientos Municipales.

\section{Bibliografía}

Brunner, Cox, Léniz, et al. (I999). Desafíos y tareas de la educación chilena al comenzar el siglo 2I, Documento del programa de educación de la Fundación Chile.

Chile, Ministerio de Educación, CANEF (2002). Objetivos fundamentales y contenidos mínimos obligatorios de la educación básica. Santiago: Autor.

Chile, Ministerio de Educación (1998). Objetivos fundamentales y contenidos mínimos obligatorios de la educación básica. Santiago: Autor.

Chile, Política Nacional de Actividad Física y Deporte. (200I). Ley del deporte 19.712. Santiago.

Cox, C. y García-Huidobro, J. (1990/I998). Visión de conjunto, La reforma educacional chilena. Madrid, 1999 (pp. 7-46). Editorial Popular.

González, M.; Guarda, S.; Molina, E. \& Santana, R. (2004). Calidad y Cantidad de la Educación Física de $\mathrm{I}^{\circ}$ a $4^{\circ}$ básico y su Contribución al Desarrollo Deportivo de la Región (Chile Deportes, Región de Magallanes y Antártica Chilena). Santiago: Universidad Metropolitana Ciencias de la Educación. Departamento de Educación Física, Deportes y Recreación.

OREALC/UNESCO (2006). "Currículo, estándares y evaluación de la calidad de la Educación”. Santiago de Chile. Doc.

Rivadeneira, María Luisa (200I). Selección y Optimización de Recursos Materiales Favorecedores del Desarrollo en la Educación Física, Consultado abril 25, 2005, en www.efdeportes.com 
Roberts, G.C.; Kleiber, D.A. y Duda, J.L. (I98I). An analysis of motivation in children's sport: The role of perceived competence in participation. Journal of Sport Psychology, 3, 206-216.

Sánchez, J.L. \& Román P.A. (2005). El Centro Escolar como Ergosistema saludable. Riesgo en Relación con los recursos Materiales y Didácticos, Espacios y Equipamiento Deportivo en Educación Física. Doc MINEDUC. 